\title{
The 100 most cited articles in prostate cancer brachytherapy: systematic review and bibliometric analysis
}

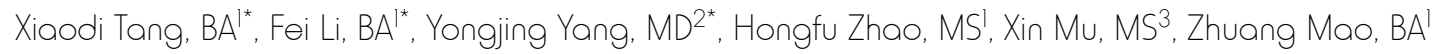 \\ 'Department of Radiation Oncology, The Third Hospital of Jilin University, Changchun, China, ${ }^{2}$ Department of Radiation Oncology, Jilin Cancer \\ Hospital, Changchun, China, ${ }^{3}$ Department of Radiation Oncology, Jilin Chemical Hospital of Chemical Industry, Jilin, China \\ "Xiaodi Tang, Fei Li, Yongjing Yang contributed equally to this work.
}

\begin{abstract}
Purpose: The aim of this study was to identify the 100 most cited research articles in prostate cancer brachytherapy (PCB) and to review the characteristics of these citation.

Material and methods: The Web of Science Core Collection was used to identify the 100 most cited articles in PCB as of December $31^{\text {st }}, 2019$. The following important information was extracted: year and month of publication, title, journal, country of origin, authors, type of article, treatment modality, and topics.

Results: The 100 most cited articles in PCB were published between 1999 and 2018, and the number of citations ranged from 455 to 54; these articles had collectively been cited 10,331 times at the time of search. These articles were from 11 countries, with most publications being from the United States $(n=61)$, followed by Canada $(n=10)$, the United Kingdom $(n=8)$, and Germany $(n=5)$. The "International Journal of Radiation Oncology, Biology, Physics" published the most articles $(n=47)$, followed by the "Journal of Urology" $(n=11)$, "Radiotherapy and Oncology" $(n=10)$, "Cancer" $(n=7)$, and "Urology" $(n=6)$. Permanent interstitial brachytherapy $(n=52)$ was the most widely used treatment modality, followed by temporary brachytherapy $(n=45)$. Disease control $(n=51)$ was the most common topic, followed by side effects $(n=44)$ and quality of life $(n=27)$.

Conclusions: The bibliometric analysis presents a detailed list of the 100 most cited articles in prostate cancer brachytherapy. There are clear recommendations for treatment with prostate cancer brachytherapy. The goal of prostate cancer brachytherapy is to improve long-term outcomes and quality of life.

J Contemp Brachytherapy 2020; 12, 3: 283-289 DOI: https://doi.org/10.5114/jcb.2020.96872
\end{abstract}

Key words: most cited articles, prostate cancer, brachytherapy, bibliometric analysis.

\section{Purpose}

Prostate cancer is the second most common cancer in men and has the fifth highest mortality rate; there are 1.3 million new cases and 359,000 related deaths every year [1]. Radical radiotherapy is an important treatment modality for prostate cancer. Brachytherapy has become an important part of radiotherapy due to its high-dose gradient $[2,3,4]$, and can be used not only as a boost to external beam radiotherapy (EBRT) $[5,6]$ but also as a monotherapy modality for localized disease $[7,8]$. At present, there are two methods for prostate brachytherapy: permanent low-dose-rate seed implantation [6,7] and temporary high-dose-rate interstitial treatment $[5,9]$. Brachytherapy can be considered a salvage treatment in men with biochemical recurrence after EBRT $[10,11]$.
The number of citations, as an objective measure, has become an important index to evaluate the influence of articles and journals. Many studies have reported the most cited articles in oncology as well as brachytherapy $[12,13,14]$. To the best of our knowledge, this report is the first to describe a bibliometric analysis of the 100 most cited articles on prostate cancer brachytherapy. The purpose of this study was to identify and describe the most cited articles in the field of brachytherapy for prostate cancer. Through analysis of the information extracted from the articles was presented and the development of prostate cancer brachytherapy was characterized.

\section{Material and methods}

Our study was a bibliometric analysis based on published articles and databases and did not require an ap- 
proval from an institutional review board. All citation count data included in the Web of Science Core Collection were used to identify eligible studies. The search strategy for prostate cancer brachytherapy articles is shown in Supplementary Table 1.

The search time ranged from 1900 to December $31^{\text {st }}$, 2019. The search results were sorted by the times cited in the Web of Science Core Collection, and the 100 most cited articles on prostate cancer brachytherapy were derived. Three articles that were not related to brachytherapy and one reprinted article, based on screening the titles and abstracts were excluded. The following four most cited articles were added. Finally, the 100 most cited articles on prostate cancer brachytherapy and the articles were ranked based on citation count identified.

A citations per year index (CPYI) was introduced to eliminate the effect, in which older studies tend to accumulate a larger number of citations [12]. The journal title, year, and month of publication, country of origin, authors, type of articles, type of brachytherapy, treatment modality, and topics were recorded. The articles types were categorized as original studies, recommendations, reviews, editorials, and surveys. For original studies, the articles were further categorized into those focusing on clinical data, physics, and radiobiology. Two independent authors performed the search, screened the articles, and extracted the information. Discrepancies were resolved by consulting a third author.

\section{Results}

The 100 most cited articles in prostate cancer brachytherapy were published from 1999 and 2018, and the number of citations ranged from 455 to 54; these articles had been collectively cited 10,331 times at the time of the search (Supplementary Table 2). The CPY index ranged from 74.40 to 2.81 .

For the 100 most cited articles, 2007 and 2009 were the most common publication years, with 10 articles each, followed by 2012, with 9 articles (Figure 1). The "International Journal of Radiation Oncology, Biology, Physics" was responsible for publishing the largest number of articles (47 articles), followed by the "Journal of Urology" with 11 articles and "Radiotherapy and Oncology" with

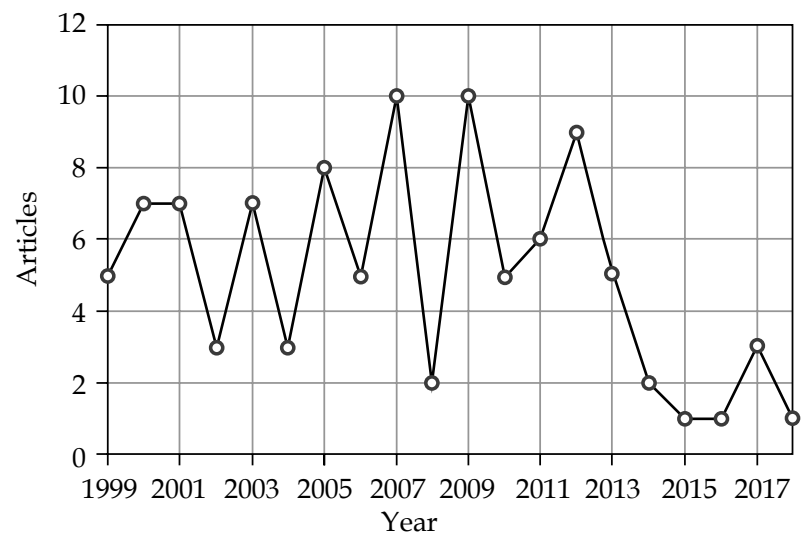

Fig. 1. The distribution by the published year for the top 100 most cited articles in brachytherapy of prostate cancer
10 articles. The remaining journals, "Cancer", "Urology”, "Journal of Clinical Oncology", and "Brachytherapy", each published seven to four articles in the top 100 cited list (Table 1).

According to the affiliation of the first author, the 100 most cited articles in prostate cancer brachytherapy were from 11 countries, with most publications being from the United States (61 articles), followed by Canada (10 articles), the United Kingdom (8 articles), Germany (5 articles), Spain, Japan, and Australia (4 articles each). The remaining countries (Switzerland, Sweden, Italy, and France) published one article each in the top 100 cited list (Table 2).

Seventy-four first authors contributed to the 100 most cited articles. Seven authors (first author) were credited with having at least three articles among the 100 most cited articles. Among these authors, Hoskin PJ and Martinez AA had five articles each, Morris WJ and Yoshioka Y had four articles each, and Morton GC, Prada PJ, and Sylvester JE had three articles each. The list of first authors, corresponding authors, and co-authors, with the largest number of publications among the 100 most cited articles is shown in Table 3.

The 100 most cited articles were categorized as original studies (91 articles), recommendations (four articles), reviews (three articles), and surveys (two articles; Table

Table 1. Journals, in which the 100 most cited prostate cancer brachytherapy articles were published

\begin{tabular}{|c|c|c|}
\hline Journal & $\begin{array}{c}\text { Number of } \\
\text { articles }\end{array}$ & $\begin{array}{l}\text { Impact factor } \\
\text { in } 2019 \\
\end{array}$ \\
\hline $\begin{array}{l}\text { International Journal of } \\
\text { Radiation Oncology, Biology, } \\
\text { Physics }\end{array}$ & 47 & 6.203 \\
\hline Journal of Urology & 11 & 5.647 \\
\hline Radiotherapy and Oncology & 10 & 5.252 \\
\hline Cancer & 7 & 6.102 \\
\hline Urology & 6 & 1.861 \\
\hline Journal of Clinical Oncology & 5 & 28.245 \\
\hline Brachytherapy & 4 & 2.030 \\
\hline BJU International & 2 & 4.524 \\
\hline $\begin{array}{l}\text { JAMA - Journal of the Ameri- } \\
\text { can Medical Association }\end{array}$ & 2 & 51.273 \\
\hline $\begin{array}{l}\text { American Journal of Clinical } \\
\text { Oncology - Cancer Clinical } \\
\text { Trials }\end{array}$ & 1 & 3.015 \\
\hline Lancet Oncology & 1 & 35.386 \\
\hline Medical Physics & 1 & 3.177 \\
\hline Radiology & 1 & 7.608 \\
\hline $\begin{array}{l}\text { Strahlentherapie } \\
\text { und Onkologie }\end{array}$ & 1 & 2.717 \\
\hline World Journal of Urology & 1 & 2.761 \\
\hline
\end{tabular}


Table 2. Countries of origin of the 100 most cited articles in the field of prostate cancer brachytherapy

\begin{tabular}{lc} 
Country & Number of articles \\
\hline The United States & 61 \\
\hline Canada & 10 \\
\hline The United Kingdom & 8 \\
\hline Germany & 5 \\
\hline Australia & 4 \\
\hline Japan & 4 \\
\hline Spain & 4 \\
\hline France & 1 \\
\hline Italy & 1 \\
\hline Sweden & 1 \\
\hline Switzerland & 1
\end{tabular}

Table 3. Number of authorships of the top 100 most frequently cited articles in the field of prostate cancer brachytherapy

\begin{tabular}{lc} 
Description & Author name (number of articles) \\
\hline Most frequent first & Hoskin PJ (5) \\
author & Martinez AA (5) \\
& Morris WJ (4) \\
Yoshioka Y (4) & Morton GC (3) \\
Prada PJ (3) & Sylvester JE (3) \\
\hline Most frequent & Martinez AA (9) \\
corresponding author & Morris WJ (5) \\
& Sylvester JE (4) \\
Yoshioka Y (4) & Hoskin PJ (3) \\
Prada PJ (3) & Williams SG (3) \\
Martinez AA (13) \\
Gustafson GS (11) \\
Grimm PD (7) \\
Blasko JC (6) \\
Host frequent co-author \\
Hotal) & Morton G (6) \\
& Bryant L (5) \\
Edmundson GK (5) \\
Gonzalez J (5) \\
McKenzie M (5) \\
Merrick G (5) \\
Morris WJ (5) \\
Ostler P (5) \\
Pai H (5) \\
Potters L (5) \\
Stock RG (5) \\
Sylvester JE (5) \\
Vicini FA (5) \\
\end{tabular}

Table 4. Type of study, treatment modality, type of radionuclide for permanent brachytherapy, follow-up time, and topics of the 100 most cited articles on prostate cancer brachytherapy

Article type Number of articles (rank in Supplementary Table 2)

\begin{tabular}{lc}
\hline Original study & 91 \\
\hline Clinic & $85(4,8,11,14,36,43,51,54$, \\
& $63,64,72,80,98) *$ \\
\hline Physics & $3(29,58,68)$ \\
\hline Radiobiology & $3(17,50,95)$ \\
\hline Recommendation & $4(1,16,18,24)$ \\
\hline Review & $3(41,77,100)$ \\
\hline Survey & $2(28,79)$ \\
\hline
\end{tabular}

Treatment modality

Permanent brachytherapy $52^{* \star}$ 125| $44^{\star \star \star}(2,8,25,33,43,54$ $60,64,65,72)$ *

\begin{tabular}{lc}
\hline${ }^{103} \mathrm{Pd}$ & $29^{* * *}(1,5,9,12,15,23,44)^{*}$ \\
\hline${ }^{131} \mathrm{Cs}$ & $3^{\text {***}}(16,77,96)$ \\
\hline
\end{tabular}

Temporary brachytherapy $\quad 45^{\star \star}(10-14,19,24,34,36$,

$42,51,55,74,81,90)$ *

Not specified 6

Combined with external beam radiotherapy

\begin{tabular}{lc}
\hline No & $65^{\text {****}(}(11,27,36,37,46,60$, \\
$63,71,90,91)^{*}$ \\
\hline Yes & $48^{* * * *}(8,10,15,47,54,62$, \\
& $67,81,84)^{*}$
\end{tabular}

Not specified

10

\begin{tabular}{lc} 
Median follow-up time (months) \\
\hline$<5$ years & $50(11,14,25,29,34,36,46$, \\
& $55,70,74,81) *$ \\
\hline 25 years & $36(2-4,7,10,15,19,23,32$, \\
& $33,44,52,65)^{*}$ \\
\hline
\end{tabular}

Not specified

14

Topic

Disease control $\quad 51^{\text {***** }}(2,5,9,10,14,19,25$,

$\frac{32,42,44,60,74){ }^{*}}{\text { Side effect }} \quad 44^{\star * * * *}(20,28,38,40,61$,
$85)$ *

\begin{tabular}{|c|c|}
\hline Quality of life & 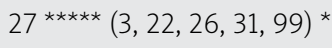 \\
\hline Cost effectiveness & $5^{* \star * * *}(12,64,75,77,88)$ \\
\hline
\end{tabular}

Catheter displacement $\quad 3^{* * * *}(29,58,68)$

Injection of hyaluronic acid

$3^{* \star * \star *}(40,66,85)$

*Only typical and educational articles are provided

**Some articles contain two treatment modalities

$* *$ Some articles contain more than one type of radionuclide

${ }_{* * * *}$ Some articles contain more than one treatment modality ${ }^{* * * * *}$ Some articles contain more than one topic 


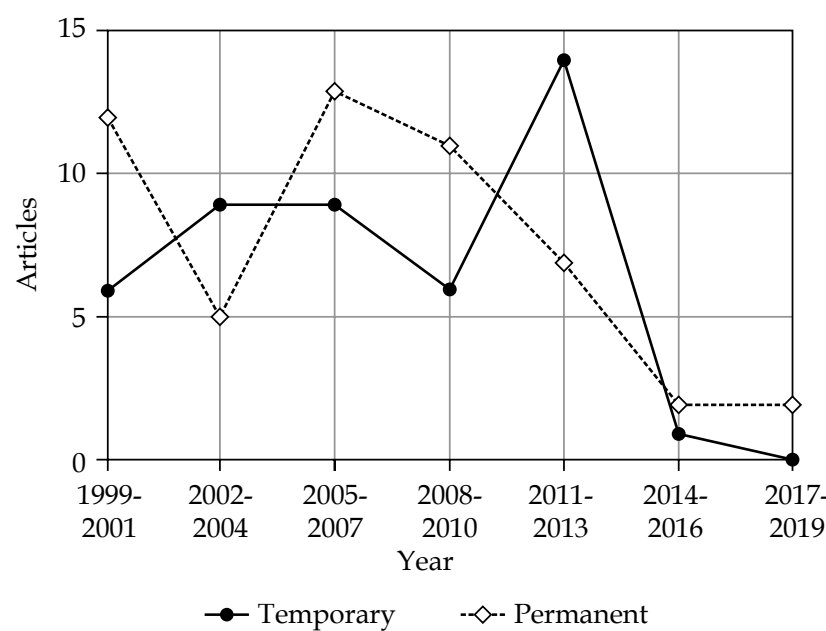

Fig. 2. The one hundred most cited articles categorized by treatment modality (temporary or permanent)

4). Iodine-125 ( $\left.{ }^{125} \mathrm{I}\right)$ and palladium-103 (103 $\left.\mathrm{Pd}\right)$ were the two most used sources for permanent brachytherapy. Of the 100 most cited articles, the number of articles with ${ }^{125} \mathrm{I}$ (44 articles) was higher than that with ${ }^{103} \mathrm{Pd}$ ( 29 articles). Disease control was the most common topic (51 articles), followed by side effects (44 articles), and quality of life (27 articles; Table 4). The treatment modality classification of the 100 most cited articles is presented in Figure 2. The radionuclide classification for permanent brachytherapy among the 100 most cited articles is demonstrated in Figure 3.

The most cited prostate cancer brachytherapy article was a recommendation from the American Brachytherapy Society (ABS) about permanent transperineal brachytherapy; this article had a high CPYI of 22.29, which ranked 6 [2]. In addition, three recommendations from the American Association of Physicists in Medicine (AAPM) [15] and the Groupe Européen de Curiethérapie - European Society for Therapeutic Radiology and Oncology (GEC) ESTRO) $[3,4]$ also received a large number of citations (153, 138 , and 123; ranked 16, 18, and 24, respectively), with high CPYIs (15.17, 9.30, and 18.92; ranked 12, 34, and 7, respectively).

\section{Discussion}

Like other citation analysis articles, this study has some limitations. Self-citations from authors or journals cannot be eliminated automatically by the Web of Science. In addition, the number of citations in the Web of Science Core Collection does not include citations by articles outside the Web of Science Core Collection. Moreover, the search expression was subjective to a certain extent. There are two other databases that provide citation count data queries. The reason for the Web of Science was chosen was explained in the previous article [12].

Patients with localized prostate cancer are the appropriate candidates for treatment with curative intent to achieve complete eradication of local tumors [16]. There are several treatment modalities, such as prostatectomy, EBRT, brachytherapy (BT) for a boost combined with EBRT $[5,17,18,19]$, or BT monotherapy $[7,8,20,21]$.

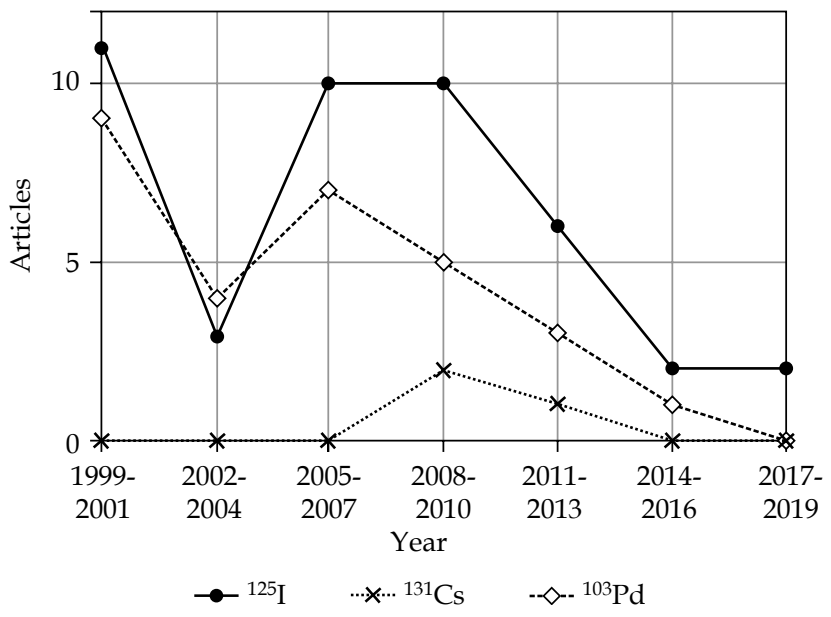

Fig. 3. The one hundred most cited articles categorized by type of radionuclide for permanent brachytherapy

In addition, brachytherapy is also a salvage treatment modality for prostate cancer after failed radiation therapy-brachytherapy, with relatively few long-term toxicities $[10,11,22,23]$. The application of various treatment modalities can follow the recommendations of an association or a society $[2,3,4,15]$. Brachytherapy is a treatment modality that delivers doses to tumors through radioactive seeds or sources placed inside the tumor. Based on the treatment time, brachytherapy can be divided into permanent brachytherapy and temporary brachytherapy.

Permanent brachytherapy, also known as radioactive seed implantation, has a long history. In 1972, Whitmore et al. [24] presented a technique for the retropubic implantation of ${ }^{125}$ I seeds in selected patients with prostate cancer; this was the basis of modern brachytherapy for prostate cancer. In 1983, Holm et al. [25] reported transperineal ${ }^{125} \mathrm{I}$ seed implantation in patients with prostate cancer guided by transrectal ultrasonography, which greatly improved the accuracy of implantation. Of the 100 most cited articles in the field of prostate cancer brachytherapy, 52 articles mentioned permanent brachytherapy. In permanent brachytherapy, radioactive seeds are indispensable. For this approach, the most important radionuclides are ${ }^{125} \mathrm{I}$ and ${ }^{103} \mathrm{Pd}$. Compared with ${ }^{125} \mathrm{I},{ }^{103} \mathrm{Pd}$ was introduced later, and its application started in 1986. There are some physical differences between ${ }^{125} \mathrm{I}$ and ${ }^{103} \mathrm{Pd}$ [2]. ${ }^{103} \mathrm{Pd}$ sources emit lower-energy photons; therefore, denser seed spacing is needed to compensate for the more rapid tissue attenuation. ABS describe the advantages and disadvantages of radionuclides in detail. However, ABS do not recommend one radionuclide to be superior to the other because of no differences in clinical outcome or side effects among any subgroups of patients in clinical studies $[2,26,27,28,29]$.

Furthermore, there are some factors that influence the choice of radionuclides. For permanent seed implantation, radioactive seeds are implanted into the prostate through a guiding needle with the guidance of a template and real-time ultrasound imaging. In theory, the guided template ensures that the guiding needles are parallel to each other, but in practice, the guiding needles will 
inevitably deviate from the standard. For ${ }^{125} \mathrm{I}$ implants, needle divergences with standard deviations of 5 degrees and 10 degrees lead to an average reduction in minimum target dose of approximately $10 \%$ and $20 \%$, respectively, and the dose coverage in the target is reduced by approximately $1 \%$ and $3 \%$, respectively. Implants designed with ${ }^{103} \mathrm{Pd}$ showed an additional $5 \%$ reduction in the minimum target dose, while the effect on dose coverage is approximately the same as that of ${ }^{125} \mathrm{I}$ implants [30]. Due to the lower initial dose rate of ${ }^{125} \mathrm{I}$, the radiation exposure to the treating staff is lower, assuming the same implantation time for both implants. However, compared with ${ }^{103} \mathrm{Pd}$, the protection time for caregivers of ${ }^{125} \mathrm{I}$ is longer due to its longer half-life (17 days vs. 59.4 days, respectively).

Evidence from clinical studies shows that prostate cancer has a low $\alpha / \beta$ ratio, so hypofractionation radiotherapy, such as high-dose-rate brachytherapy (HDR-BT), may be more effective for prostate cancer [31,32,33,34]. HDR brachytherapy uses a single radioactive source with high activity that is programmed to send and retract back to each implanted needle in sequence via a remote control. Compared with permanent seed implantation (PSI), HDRBT has many advantages. In terms of radiation safety, HDR-BT does not expose the medical staff because the implantation of applicators and the dose delivery are separated in time and space. Moreover, the patients are not radioactive when they return home because the radiation source is retracted into the afterloading needle after HDR-BT treatment. In PSI, the actual delivered dose to the tissue is related to post-operative seed distribution. With post-operative edema or subsequent tumor regression, the distribution of seeds will change continuously, which will affect the dose distribution. HDR significantly improves the radiation dose distribution due to the precise control of source dwell position and the optimization of dwell time during the treatment. For fractionated HDR brachytherapy, within one application, a reliable high-quality catheter fixation method is needed to avoid displacement of the catheters and movement of the gland [35,36]. Pretreatment imaging is critical to evaluate the catheters or to correct the catheters for large migrations, thereby ensuring the treatment quality [37]. More importantly, HDR brachytherapy as prostate cancer monotherapy has less toxicity than permanent seed implantation [38,39].

Compared with PSI, HDR-BT can significantly reduce the cost of treatment [38]. The cost of HDR-BT was 19\% lower than that of PSI, on average. The reduction in HDR treatment-related costs is mainly because it does not need to purchase radioactive sources. According to a randomized clinical trial, the relapse-free survival of patients with localized prostate cancer who received EBRT combined with an HDR brachytherapy boost was better than that of patients who received EBRT alone, with similar severe late urinary and bowel morbidities [5].

Through cost-effectiveness analysis, Charles found that a brachytherapy boost significantly reduced the expected lifetime treatment costs, $\$ 68,696$ vs. $\$ 114,944$, and the brachytherapy boost had an expected quality-adjusted life years of 10.8 years, compared to 9.3 years for intensity-modulated radiation therapy (IMRT) alone [40]. Therefore, IMRT combined with an HDR brachytherapy boost is a more cost-effective treatment for intermediateto high-risk prostate cancer than IMRT alone. EBRT combined with a brachytherapy boost has achieved good clinical outcomes, and in low- and intermediate-risk prostate cancer, brachytherapy as monotherapy has accomplished good results [7,20,39,41]. Moreover, compared with EBRT, both LDR and HDR have improved cost-effectiveness [42].

Six authors (including as co-authors) were credited with having at least six articles among the 100 most cited articles. Among these authors, at the time of publication, Martinez AA and Gustafson GS were from the William Beaumont Hospital, Oakland University, MI, USA, with 13 and 11 articles, respectively. Grimm PD and Blasko JC were from the Seattle Prostate Institute, WA, USA, and had seven and six articles, respectively. Hoskin PJ and Morton $G$ were from the Mount Vernon Cancer Centre, Northwood, United Kingdom and the Odette Cancer Centre, Toronto, Canada, respectively, and they both had six articles. It can be seen from the concentrated authors' distribution that the above four institutions have made the greatest contribution to brachytherapy for prostate cancer and are leading in the development of new technologies.

A steep dose gradient is one of the most important characteristics of brachytherapy, so the distribution of guiding needles for PSI or catheters for HDR is particularly important. The quality of implantation directly determines the quality of dose distribution, including control of the hot and cold spots in the tumor and the protection of urethra and adjacent organs at risk (OARs). The distance between the surface of adjacent OARs and the outer border of tumor directly determines the dose delivered. To increase this distance, hyaluronic acid (HA) is injected into the perirectal fat to increase the distance between the prostate and the anterior wall of rectum, thereby reducing the dose to the rectum $[43,44,45]$. This is a novel method, and the patients tolerated the implantation procedure very well with minimal discomfort. For an HDR boost dose of $1150 \mathrm{cGy}$, the mean maximum dose $\left(D_{\max }\right)$ to the rectum decreased from $708 \mathrm{cGy}$ to $507 \mathrm{cGy}$ [43]. Patients treated with ${ }^{125} \mathrm{I}$ brachytherapy and an injection of HA had a significantly lower incidence of mucosal damage on proctoscopic examinations (5\% vs. $36 \%$, $p=0.002)$ and macroscopic rectal bleeding ( $0 \%$ vs. $12 \%$, $p=0.047)$ than those treated with ${ }^{125} \mathrm{I}$ brachytherapy alone without HA [44].

In general, brachytherapy for prostate cancer has achieved satisfactory long-term clinical results $[6,7,17$, 46,47]. Because of its promising efficacy, brachytherapy for prostate cancer focuses on long-term follow-up results. Of the 100 most cited articles, 36 articles had a median follow-up time of more than 5 years. Moreover, based on ensuring the curative effect, reduction of side effects and quality of life improvement are also two important subjects. Of the 100 most cited articles, 44 and 27 articles involved side effects and quality of life, respectively. These results suggest that we should pay more attention to these important issues in future research.

The significance of this study is that it listed the most cited articles and conducted a bibliometric analysis. Although citation number is not the only standard for a good paper, the total number of citations is an objective 
evaluation index of paper quality to some extent. This paper provides some references for medical staff engaged in brachytherapy for prostate cancer, especially for those with less than 5 years of experience, in the selection of treatment mode, treatment technology, etc.

\section{Conclusions}

The bibliometric analysis presents a detailed list of the 100 most cited articles in prostate cancer brachytherapy. There are clear recommendations for treatment with prostate cancer brachytherapy. The goal of prostate cancer brachytherapy is to improve long-term outcomes and quality of life.

\section{Disclosure}

The authors report no conflict of interest.

Supplemental Tables are available online at the journal homepage.

\section{References}

1. Bray F, Ferlay J, Soerjomataram I et al. Global cancer statistics 2018: GLOBOCAN estimates of incidence and mortality worldwide for 36 cancers in 185 countries. CA Cancer J Clin 2018; 68: 394-424.

2. Nag S, Beyer D, Friedland J et al. American Brachytherapy Society (ABS) recommendations for transperineal permanent brachytherapy of prostate cancer. Int J Radiat Oncol Biol Phys 1999; 44: 789-799.

3. Kovács G, Pötter R, Loch T et al. GEC/ESTRO-EAU recommendations on temporary brachytherapy using stepping sources for localised prostate cancer. Radiother Oncol 2005; 74: 137-148.

4. Hoskin PJ, Colombo A, Henry A et al. GEC/ESTRO recommendations on high dose rate afterloading brachytherapy for localised prostate cancer: An update. Radiother Oncol 2013; 107: 325-332.

5. Hoskin PJ, Rojas AM, Bownes PJ et al. Randomised trial of external beam radiotherapy alone or combined with highdose-rate brachytherapy boost for localised prostate cancer. Radiother Oncol 2012; 103: 217-222.

6. Potters L, Morgenstern C, Calugaru E et al. 12-year outcomes following permanent prostate brachytherapy in patients with clinically localized prostate cancer. J Urology 2005; 173: 1562-1566.

7. Grimm PD, Blasko JC, Sylvester JE et al. 10-year biochemical (prostate-specific antigen) control of prostate cancer with I-125 brachytherapy. Int J Radiat Oncol Biol Phys 2001; 51: 31-40.

8. Martinez AA, Pataki I, Edmundson G et al. Phase II prospective study of the use of conformal high-dose-rate brachytherapy as monotherapy for the treatment of favorable stage prostate cancer: A feasibility report. Int J Radiat Oncol Biol Phys 2001; 49: 61-69.

9. Demanes DJ, Rodriguez RR, Schour L et al. High-dose-rate intensity-modulated brachytherapy with external beam radiotherapy for prostate cancer: California endocurietherapy's 10-year results. Int J Radiat Oncol Biol Phys 2005; 61: 1306-1316.

10. Grado GL, Collins JM, Kriegshauser JS et al. Salvage brachytherapy for localized prostate cancer after radiotherapy failure. Urology 1999; 53: 2-10.

11. Burri RJ, Stone NN, Unger P et al. Long-term outcome and toxicity of salvage brachytherapy for local failure after initial radiotherapy for prostate cancer. Int J Radiat Oncol Biol Phys 2010; 77: 1338-1344.

12. Tang $\mathrm{X}$, Zhao $\mathrm{Z}$, Zhao $\mathrm{H}$ et al. The 100 most cited articles in cervical cancer brachytherapy. Brachytherapy 2020; 19: 181-193.

13. De la Garza-Ramos R, Benvenutti-Regato M, Caro-Osorio E. The 100 most-cited articles in spinal oncology. J Neurosurg Spine 2016; 24: 810-823.

14. Atci IB, Yilmaz H, Samanci MY. The top 50 most-cited articles on low-grade glioma: a bibliometric analysis. Brit J Neurosurg 2019; 33: 171-175.

15. Nath R, Bice WS, Butler WM et al. AAPM recommendations on dose prescription and reporting methods for permanent interstitial brachytherapy for prostate cancer: Report of Task Group 137. Med Phys 2009; 36: 5310-5322.

16. Valerio M, Ahmed HU, Emberton $\mathrm{M}$ et al. The role of focal therapy in the management of localised prostate cancer: a systematic review. Eur Urol 2014; 66: 732-751.

17. Sylvester JE, Grimm PD, Blasko JC et al. 15-year biochemical relapse free survival in clinical Stage T1-T3 prostate cancer following combined external beam radiotherapy and brachytherapy; Seattle experience. Int J Radiat Oncol Biol Phys 2007; 67: 57-64.

18. Kestin LL, Martinez AA, Stromberg JS et al. Matched-pair analysis of conformal high-dose-rate brachytherapy boost versus external-beam radiation therapy alone for locally advanced prostate cancer. J Clin Oncol 2000; 18: 2869-2880.

19. Spratt DE, Zumsteg ZS, Ghadjar P et al. Comparison of highdose (86.4 Gy) IMRT vs combined brachytherapy plus IMRT for intermediate-risk prostate cancer. BJU Int 2014; 114: 360-367.

20. Demanes DJ, Martinez AA, Ghilezan M et al. High-dose-rate monotherapy: safe and effective brachytherapy for patients with localized prostate cancer. Int J Radiat Oncol Biol Phys 2011; 81: 1286-1292.

21. Henry AM, Al-Qaisieh B, Gould K et al. Outcomes following iodine-125 monotherapy for localized prostate cancer: the results of leeds 10-year single-center brachytherapy experience. Int J Radiat Oncol Biol Phys 2010; 76: 50-56.

22. Beyer DC. Permanent brachytherapy as salvage treatment for recurrent prostate cancer. Urology 1999; 54: 880-883.

23. Chen $C P$, Weinberg V, Shinohara $K$ et al. Salvage HDR brachytherapy for recurrent prostate cancer after previous definitive radiation therapy: 5-year outcomes. Int J Radiat Oncol Biol Phys 2013; 86: 324-329.

24. Whitmore Jr WF, Hilaris B, Grabstald H. Retropubic implantation of Iodine 125 in the treatment of prostatic cancer. J Urol 1972; 108: 918-920.

25. Holm HH, Juul N, Pedersen JF et al. Transperineal 125iodine seed implantation in prostatic cancer guided by transrectal ultrasonography. J Urol 2002; 167: 985-988.

26. Wallner K, Merrick G, True L et al. I-125 versus Pd-103 for low-risk prostate cancer: Preliminary PSA outcomes from a prospective randomized multicenter trial. Int J Radiat Oncol Biol Phys 2003; 57: 1297-1303.

27. Peschel RE, Colberg JW, Chen $Z$ et al. Iodine 125 versus palladium 103 implants for prostate cancer: Clinical outcomes and complications. Cancer J 2004; 10: 170-174.

28. Kollmeier MA, Pei X, Algur E et al. A comparison of the impact of isotope ((125)I vs. (103)Pd) on toxicity and biochemical outcome after interstitial brachytherapy and external beam radiation therapy for clinically localized prostate cancer. Brachytherapy 2012; 11: 271-276.

29. Zhang LL, Ma L, Tian JH et al. (125)I versus (103)Pd brachytherapy for low risk prostate cancer: a systematic review. Ai Zheng 2009; 28: 872-878.

30. Nath S, Chen Z, Yue N et al. Dosimetric effects of needle divergence in prostate seed implant using I-125 and Pd-103 radioactive seeds. Med Phys 2000; 27: 1058-1066. 
31. Wang JZ, Li XA, Yu CX et al. The low alpha/beta ratio for prostate cancer: What does the clinical outcome of HDR brachytherapy tell. Int J Radiat Oncol Biol Phys 2003; 57: 1101-1108.

32. Wang JZ, Guerrero M, Li XA. How low is the alpha/beta ratio for prostate cancer? Int J Radiat Oncol Biol Phys 2003; 55: 194-203.

33. Kal HB, Van Gellekom MPR. How low is the alpha/beta ratio for prostate cancer? Int J Radiat Oncol Biol Phys 2003; 57: 1116-1121.

34. King CR, Fowler JF. A simple analytic derivation suggests that prostate cancer alpha/beta ratio is low. Int J Radiat Oncol Biol Phys 2001; 51: 213-214.

35. Hoskin PJ, Bownes PJ, Ostler P et al. High dose rate afterloading brachytherapy for prostate cancer: catheter and gland movement between fractions. Radiother Oncol 2003; 68: 285-288.

36. Damore SJ, Syed AMN, Puthawala AA et al. Needle displacement during HDR brachytherapy in the treatment of prostate cancer. Int J Radiat Oncol Biol Phys 2000; 46: 1205-1211.

37. Simnor T, Li S, Lowe $\mathrm{G}$ et al. Justification for inter-fraction correction of catheter movement in fractionated high doserate brachytherapy treatment of prostate cancer. Radiother Oncol 2009; 93: 253-258.

38. Grills IS, Martinez AA, Hollander $M$ et al. High dose rate brachytherapy as prostate cancer monotherapy reduces toxicity compared to low dose rate palladium seeds. J Urology 2004; 171: 1098-1104.

39. Martinez AA, Demanes J, Vargas C et al. High-Dose-Rate Prostate Brachytherapy An Excellent Accelerated-Hypofractionated Treatment for Favorable Prostate Cancer. Am J Clin Oncol 2010; 33: 481-488.

40. Vu CC, Blas KG, Lanni TB et al. Cost-effectiveness of prostate boost with high-dose-rate brachytherapy versus intensity-modulated radiation therapy in the treatment of intermediate-high risk prostate cancer. Brachytherapy 2018; 17: 852-857.

41. Morris WJ, Keyes M, Spadinger I et al. Population-based 10-year oncologic outcomes after low-dose-rate brachytherapy for low-risk and intermediate-risk prostate cancer. Cancer 2013; 119: 1537-1546.

42. Shah C, Lanni TB, Ghilezan MI et al. Brachytherapy provides comparable outcomes and improved cost-effectiveness in the treatment of low/intermediate prostate cancer. Brachytherapy 2012; 11: 441-445.

43. Prada PJ, Fernandez J, Martinez AA et al. Transperineal injection of hyaluronic acid in anterior perirectal fat to decrease rectal toxicity from radiation delivered with intensity modulated brachytherapy or ebrt for prostate cancer patients. Int J Radiat Oncol Biol Phys 2007; 69: 95-102.

44. Prada PJ, Gonzalez H, Menendez C et al. Transperineal injection of hyaluronic acid in the anterior perirectal fat to decrease rectal toxicity from radiation delivered with low-doserate brachytherapy for prostate cancer patients. Brachytherapy 2009; 8: 210-217.

45. Prada PJ, Jimenez I, Gonzalez-Suarez H et al. High-dose-rate interstitial brachytherapy as monotherapy in one fraction and transperineal hyaluronic acid injection into the perirectal fat for the treatment of favorable stage prostate cancer: Treatment description and preliminary results. Brachytherapy 2012; 11: 105-110.

46. Galalae RM, Kovacs G, Schultze J et al. Long-term outcome after elective irradiation of the pelvic lymphatics and local dose escalation using high-dose-rate brachytherapy for locally advanced prostate cancer. Int J Radiat Oncol Biol Phys 2002; 52: 81-90.

47. Taira AV, Merrick GS, Butler WM et al. Long-term outcome for clinically localized prostate cancer treated with perma- nent interstitial brachytherapy. Int I Radiat Oncol Biol Phys 2011; 79: 1336-1342. 